\title{
二元共组装法制备无裂痕反蛋白石结构薄膜的研究
}

\author{
罗文吴 ${ }^{a} \quad$ 朱水洪 $^{a} \quad$ 林友辉*, ${ }^{a}$ 刘向阳*,,$b$ \\ $\left({ }^{a}\right.$ 厦门大学材料学院 物理科学与技术学院 生物仿生及软物质研究院 \\ 福建省柔性功能材料重点实验室 厦门 361005) \\ ( ${ }^{b}$ 新加坡国立大学物理系 新加坡 117542)
}

\begin{abstract}
摘要 近年来, 由于在光学、电学和生化等领域具有广泛的潜在应用, 有序多孔反蛋白石结构薄膜的研究引起了人们 的广泛关注. 但是其在制备过程中常常会形成一些无法控制的缺陷，限制了这类材料的普及和实际应用. 通过使用两 种基质前驱体(正硅酸乙醌或丝素蛋白)与胶体小球混合共组装, 探究了二元体系共组装法制备无裂痕反蛋白石结构薄 膜的可行性. 并用扫描电镜和可见光谱对薄膜结构进行了表征. 结果表明, 对于正硅酸乙酯体系, 在不影响胶体小球 有序排列的条件下，正硅酸乙酯在小球间的空隙中发生溶胶凝胶转变，与微球共同组装成有序致密的整体，去除微球 模板后，可以得到大规模 $(>200 \mu \mathrm{m})$ 无缺陷有序的反蛋白石结构薄膜. 而对于大分子丝素蛋白体系，由于它和胶体小球 有较强的相互作用力, 会抑制胶体小球的有序组装，导致无法形成有序结构薄膜. 对两种二元共组装体系进行了实验 探索, 实验结果不仅有助于人们了解共组装方式的适用范围, 而且为设计和制备无缺陷反蛋白石薄膜提供了新的途径. 关键词 共组装; 反蛋白石结构; 无裂痕; 胶体小球
\end{abstract}

\section{Preparation of Crack-free Inverse-opal Films by Template/Matrix Co-assembly}

\author{
Luo, Wenhao $^{a} \quad$ Zhu, Shuihong ${ }^{a} \quad$ Lin, Youhui ${ }^{* a} \quad$ Liu, Xiang Yang ${ }^{* a, b}$ \\ $\left({ }^{a}\right.$ College of Materials \& College of Physical Science and Technology, Research Institute for Biomimetics and Soft Matter, \\ Fujian Provincial Key Laboratory for Soft Functional Materials Research, Xiamen University, Xiamen 361005) \\ ( ${ }^{b}$ Department of Physics, National University of Singapore, Singapore 117542)
}

\begin{abstract}
Recently, there has been a significant interest in utilizing well-ordered, porous inverse-opal films for applications in optical, electronic and (bio)chemical fields. However, uncontrolled defects are always formed during their preparation process, which limit their practical applications. In this work, we examine the feasibility of using template/matrix co-assembly strategies to fabricate crack-free inverse opal thin films. Polystyrene spheres (PS) are chosen as a colloidal template, and two matrix precursors [tetraethoxysilane (TEOS) precursor and regenerated silk fibroin solution] are used for the current study. Our scanning electron microscope (SEM) and optical spectrum results show that, for the TEOS-based system, the resulting silica gel due to the sol-gel transition of TEOS can effectively fill the gap between particles, but cannot affect the self-assembly of PS colloidal particles. After selective removal of the PS template, centimeter-scale crack-free and well-ordered inverse opal films can be obtained. In addition, for a constant concentration of TEOS, the film thickness and order degree of structure can be simply tuned by adjusting the concentrations of colloidal spheres. In comparison with indirect approach through template self-assembly and liquid infiltration, such a co-assembly approach can effectively minimize the associated cracking and avoid the need for matrix infiltration into the preassembled colloidal spheres. On the other hand, macro-molecule silk fibroin has a relatively strong interaction with PS colloidal particles, which is demonstrated by SEM and confocal images. Due to their interaction, silk fibroin molecules are preferably adsorbed on the surface of PS spheres, which can restrain the self-assembly of colloidal particles. As a result, it cannot form well-ordered silk film based on such co-assembly strategy. That is to say, the co-assembly approach is not suitable for systems that matrices have strong interactions with templates. These findings pave the way to use the template/matrix co-assembly strategy for rationally designing and developing crack-free inverse opal films and to apply such well-ordered and porous materials in a variety of fields.

Keywords co-assembly; inverse-opal structure; crack-free; colloidal spheres
\end{abstract}

\footnotetext{
*E-mail: linyouhui@xmu.edu.cn; phyliuxy@nus.edu.sg

Received May 31, 2017; published July 3, 2017.

Project supported by the National Natural Science Foundation of China (Nos. 21401154, U1405226), the 111 Project (No. B16029), the Natural Science Foundation of Guangdong Province (2014A030310005) and the Fundamental Research Funds for the Central Universities of China (No. 20720170011). 项目受国家自然科学基金(Nos. 21401154, U1405226)、111 计划(No. B16029)、广东省自然科学基金(No. 2014A030310005)及厦门大学校长基金(No. 20720170011)资助.
} 


\section{1 引言}

自组装技术作为一种成本低、易操作的工艺, 在制 备具有纳米微孔结构的固体材料上有着极大的优势 ${ }^{[1]}$. 其中, 通过将胶体小球组装模板进行浇筑复刻得到的反 蛋白石多孔材料尤其受到人们的普遍关注. 这是因为反 蛋白石结构可以通过胶体自组装的方式获得多层次的、

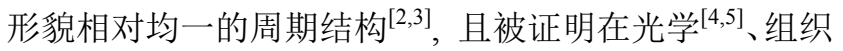
工程 ${ }^{[6]}$ 、传感器 ${ }^{[7]}$ 等领域有着重要的应用.

尽管常规的自组装方式能得到有序的反蛋白石结 构薄膜, 但是其符合严格周期排列, 有序无缺陷的区域 较小, 通常长度不超过 $10 \mu \mathrm{m}^{[8]}$. 这是由于通过自组装 方法得到的反蛋白石总是会产生随机的缺陷或裂 痕 ${ }^{[9,10]}$, 而有缺陷裂痕的反蛋白石结构会降低整个材料 的稳定性, 致使光学和力学性质下降, 从而限制了自组 装方法得到的有序多孔薄膜的应用范围. 针对这一问 题，人们近年来开发了各种不同的新方法来可控制备大 面积有序多孔薄膜 ${ }^{[11]}$.

二元或者多元体系的组装一直是化学领域研究的 热点, 通过适当的选取两种或者多种材料直接混合, 再 利用一定的成型方式, 就有机会直接得到多功能结构有 序的复合材料, 例如, 功能化的纤维 ${ }^{[12]}$ 、薄膜 ${ }^{[13,14]}$ 和气 凝胶 ${ }^{[15]}$. 迄今, 直接用共组装制备光子晶体反蛋白石结 构的方式相对较少. 将基质材料的前驱体溶液与胶体小 球混合后共同组装，直接得到已被基质材料填充好的有 序光子晶体模板, 有望获得完美无裂痕的反蛋白石结构 薄膜. 本工作分别以正硅酸乙酯(TEOS)和再生丝素蛋 白溶液为前驱体, 与聚苯乙烯微球共混, 在玻璃片上进 行垂直沉降, 得到二氧化硅与胶体小球的共组装体和丝 素蛋白与胶体小球的共组装体, 再分别用高温传烧和溶 剂溶解的方式去除胶体模板, 得到多孔的二氧化硅和丝 素蛋白薄膜. 最后, 用扫描电镜和透反显微镜等对所形 成的组装结构及其光学性质进行了表征.

\section{2 结果与讨论}

\section{1 制备反蛋白石薄膜的常规方法}

反蛋白石薄膜通常用牺牲模板法得到, 该方法有三 个步骤: (1)使胶体小球预先组装成一个密堆积的模板; (2)将胶体小球的模板浸润到可以形成固体的前驱体母 相溶液中; (3)待浸润的溶液固化后, 选择性地去除胶体 模板从而生成反蛋白石结构 ${ }^{[16,17]}$.

如图 1 所示, 运用该方法无论对于能水解固化的小 分子, 如正硅酸乙酯, 还是对于能自组装交联的生物大 分子蚕丝丝素蛋白, 均能得到相对规整的反蛋白石结 构. 但对于样品整体来说, 如图 2 所示, 这样的制备方 法会产生大量的缺陷 ${ }^{[9]}$, 这是由于胶体小球的模板在干 燥过程中, 水分的挥发会在小球之间的空隙中产生应 力, 从而产生裂纹 ${ }^{[18,19]}$. 缺陷的产生极大地限制了反蛋
白石结构薄膜的应用, 为解决这一问题, 有许多研究讨 论了自组装的动力学机理并尝试了多种优化方式 ${ }^{[20 ~ 22], ~}$ 但到现在为止，仍然没有一个相对可靠的、成本较低的、 容易普及的制备大面积(厘米级别)多层无缺陷反蛋白石 结构薄膜的方法.

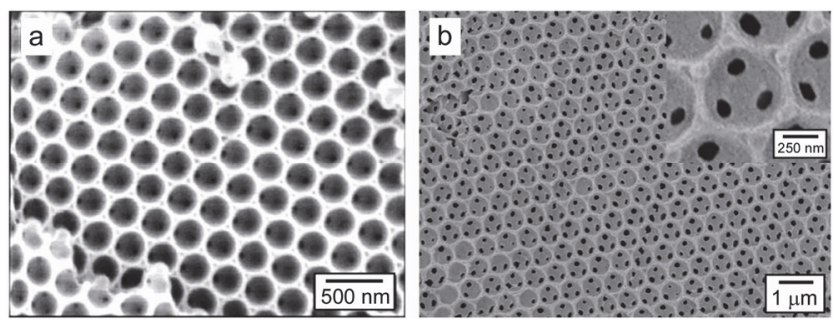

图 1 使用牺牲模版法制备的反蛋白石结构薄膜局部排列规整的区域 Figure 1 Crack-less domain of Inverse opal structure fabricated by sacrificing colloidal template

(a) $\mathrm{SiO}_{2}$ inverse opal structure ${ }^{[16]}$, Copyright 2002, American Chemical Society. (b) silk fibroin inverse opal structure ${ }^{[17]}$, Copyright 2013, Wiley-VCH
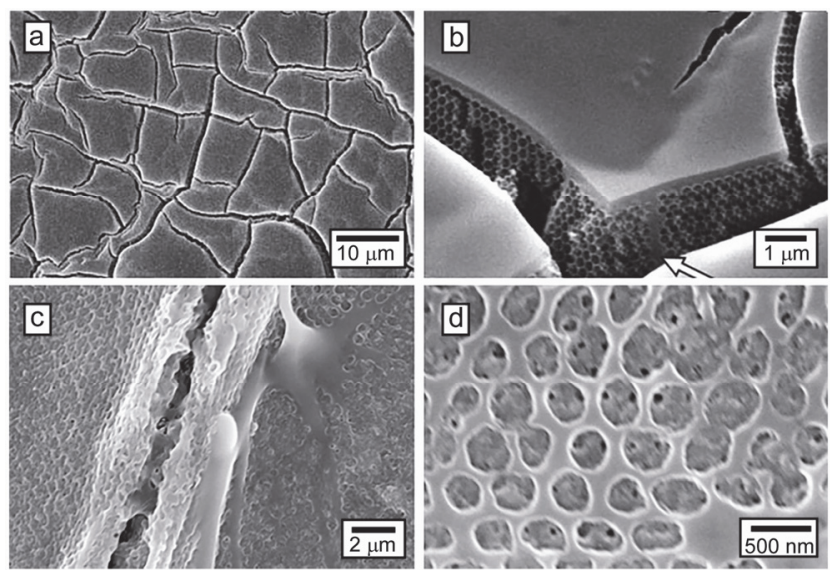

图 2 使用牺牲模版法制备得到的反蛋白石结构薄膜大范围有裂痕的 区域

Figure 2 Crack and flows at inverse opal structures fabricated by sacrificing colloidal samples

(a, b) $\mathrm{SiO}_{2}$ inverse opal structure ${ }^{[8]}$, Copyright 2010, Proceedings of the National Academy of Sciences. (c, d) silk fibroin inverse opal structure

\section{2 二氧化硅反蛋白石结构薄膜的形貌表征}

为解决前述问题, 我们尝试使用了共组装的方法制 备二氧化硅反蛋白石结构. 图 3 为通过正硅酸乙酯与 PS 微球的共组装得到的二氧化硅反蛋白石结构薄膜. 从图 $3 \mathrm{a} \sim 3 \mathrm{c}$ 中可以看到, 通过共组装方法能够得到大尺度 (长度 $>200 \mu \mathrm{m})$ 严格周期排列, 无缺陷的二氧化硅反蛋 白石结构薄膜, 远超常规制备方式所得到的无缺陷区域 (一般长度不超过 $10 \mu \mathrm{m}$ ). 这表明正硅酸乙酯与 PS 微球 混合后进行的共组装相比 PS 微球单独的自组装能抑制 大范围缺陷的产生.

对于含有同一浓度 TEOS 前驱体的共混溶液, 调整 胶体小球的浓度能显著改变后续共组装所形成薄膜的 厚度和有序程度. 如图 4a 所示, 当在混合溶液中加入的 TEOS 前驱体溶液含量为固定的 $0.15 \mathrm{~mL}$ 时, 调整胶体 


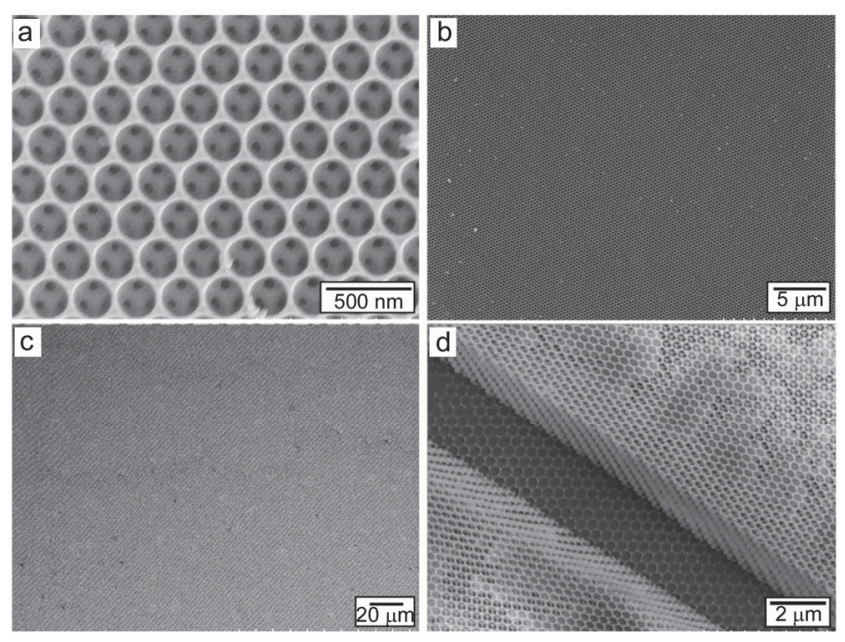

图 3 共自组装的方式得到的大面积规整的二氧化硅反蛋白石结构的 SEM 图像

Figure 3 SEM images of $\mathrm{SiO}_{2}$ inverse opal structures obtained by co-assembly process

(a) High magnification and low magnification, (b, c) SEM images of crack-free inverse-opal films, (d) the sectional view of inverse opal film

小球的浓度, 最终得到的薄膜在宏观上对光的反射效果 有明显的差别, 这意味着 PS 小球的浓度和 TEOS 前驱 体溶液的浓度必须符合一定的比例才能达到较好的效 果. 图 $4 \mathrm{~b} \sim 4 \mathrm{e}$ 进一步展示了对于同一浓度的 TEOS 前驱
体与不同浓度小球共组装后得到的薄膜在厚度和结构 有序度上的差别. 如图 $4 \mathrm{~b}$ 所示, 所得薄膜的厚度与小球 的浓度线性相关. 而通过不同条件下反蛋白石结构的微 观形貌可以看出只有当 PS 小球的浓度控制在适当的范 围时, 薄膜才表现出最佳的结构有序度. 如图 $4 \mathrm{e}$ 所示, 当胶体小球的浓度过低时，在薄膜上一些本应被小球占 据的空间会被水解得到的二氧化硅完全填充，从而形成 一个类似 “补丁” 的区域，防止了裂痕的产生; 而当小 球的浓度较高时, 随着薄膜整体厚度的增加, 薄膜最终 仍然会形成沿着小球取向的三角形裂纹, 如图 4c 所示. 我们推测, 这种形状裂纹的形成是由于填充在小球间隙 中的二氧化硅不能完全消除干燥过程中所产生的应力, 而随着薄膜厚度的增长, 成型过程中积累的应力就会增 多, 当应力积累到一定大小后就会导致裂纹的产生. 当 胶体小球的浓度与 TEOS 匹配时, 则能获得大面积的无 裂痕反蛋白石结构薄膜, 如图 $4 \mathrm{~d}$ 所示.

接着, 我们还用光纤光谱仪观察对比了大面积无缺 陷反蛋白石结构的光子晶体和有大量缺陷的反蛋白石 光子晶体的反射光谱. 从图 5 可以看到, 对于同一光源, 在对应光子禁带波段上 ${ }^{[23]}$, 具有规整结构的反蛋白石 薄膜相比于有大量缺陷的反蛋白石薄膜有着更强的反 射光信号. 除此以外, 二者的反射峰也不太一致, 我们 推测这是由于胶体微球在组装过程中产生缺陷，导致整
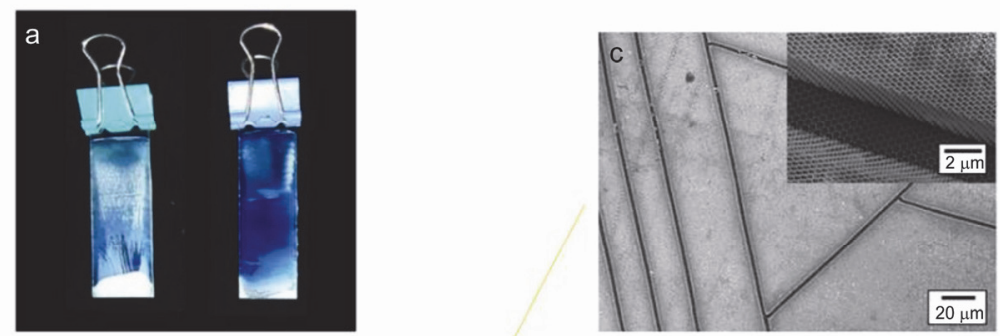

PS concentration

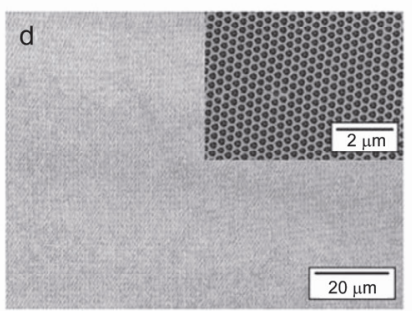

$0.2 w t \%$

PS concentration: $\quad 0.2 w t \% \quad 0.125 w t \%$

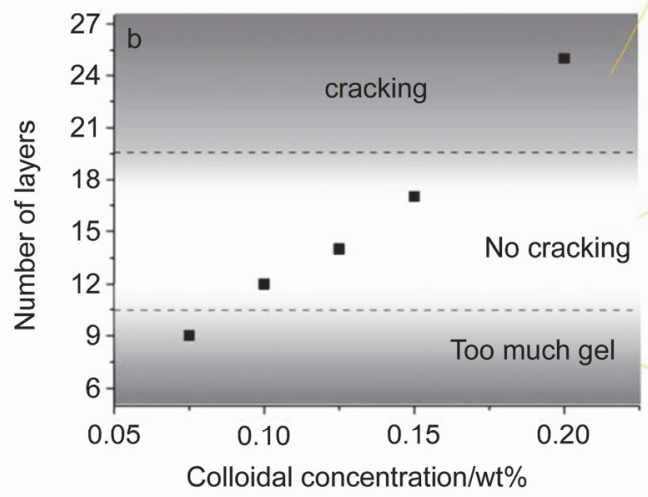

$0.125 w t \%$

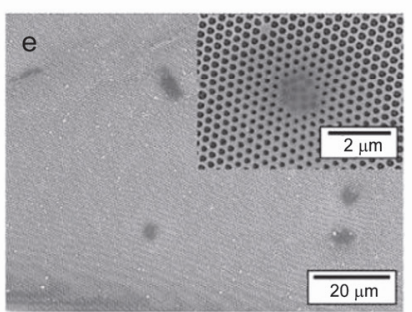

$0.075 w t \%$

图 4 (a)垂直沉降得到的二氧化硅反蛋白石光子晶体宏观图像、(b)通过 SEM 截面表征得到的光子晶体层数和胶体小球浓度的关系图(可以看到 小球浓度需要保持在一定范围才能最大限度的避免缺陷)及(c e) 不同胶体小球浓度对应的微观形貌

Figure 4 (a) Optical photography of the $\mathrm{SiO}_{2}$ inverse opal films, (b) Number of layers of photonic crystal changed along with the particle concentration (particle numbers should be limited into a small range in order to prevent flaws) and $(\mathrm{c} \sim \mathrm{e})$ different morphology of $\mathrm{SiO}_{2}$ inverse opal structure with different colloidal concentration 
个光子晶体薄膜的排列周期发生了变化(间距变大, 二 氧化硅组分增加), 进而导致有大量缺陷的反蛋白石薄 膜峰位发生了红移. 根据布拉格定律 ${ }^{[24]}$.

$$
\begin{aligned}
& \lambda_{\max }=2 d_{111} n_{\mathrm{eff}} \sin \theta \\
& n_{\mathrm{eff}}{ }^{2}=f n_{0}{ }^{2}+(1-f) n_{\mathrm{c}}{ }^{2} \\
& d_{111}=\sqrt{2 / 3} D
\end{aligned}
$$

其中 $\lambda_{\text {max }}$ 是光谱的最高反射峰位; $d_{111}$ 是晶格周期常数, 其数值等价为 $\sqrt{2 / 3} D ; n_{\mathrm{eff}}$ 是反蛋白石结构的等效折射 率; $\theta$ 是光源的入射角; $n_{\mathrm{c}}$ 和 $n_{0}$ 分别是二氧化硅的折射率 和空气的折射率; $D$ 是制备反蛋白石结构过程中所用到 的胶体微球的直径; $f$ 是光子晶体的填充系数, 它反映了 光子晶体中不同组分所占的比例. 对于反蛋白石结构的 光子晶体而言, 当有缺陷生成时, 二氧化硅在单位体积 内会填充更多的空间, 从而提高 $n_{\mathrm{eff}}$ 的数值, 进而会使 最终反射光谱的峰位发生红移. 综上, 反射强度和峰位 置改变都进一步证明, 通过共组装方式, 可以有效减少 反蛋白石结构薄膜中的缺陷, 能显著提高该薄膜作为光 子晶体的反射效率.

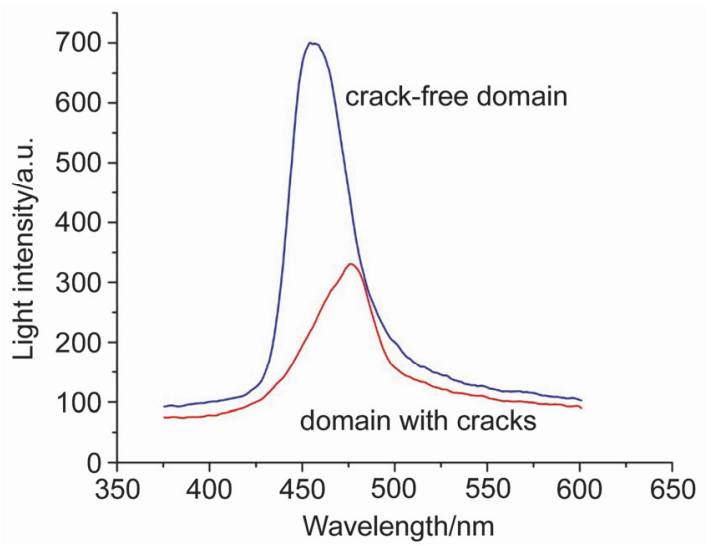

图 5 有缺陷的二氧化硅反蛋白石结构和无缺陷的二氧化硅反蛋白石 结构的反射光谱图

Figure 5 Optical spectrum of crack-free inverse-opal structure and the same structure with cracks

\section{3 丝素蛋白反蛋白石结构薄膜的形貌表征}

除了 TEOS 前驱体与胶体小球的共组装体系外, 我 们还尝试了大分子丝素蛋白与胶体微球的共组装体系. 图 6 显示了丝素蛋白与胶体微球共组装后的微观形貌. 从图中可以看出二者的结合并没有形成有序的结构, 相 反, 几乎所有的胶体小球都没有进行有序的排列, 由此 说明大分子丝素蛋白并不适用于共组装法来制备反蛋 白石结构. 从图 6a 中可以看到, 小球的表面附着上了一 层丝素蛋白, 且在后续使用四氢呋喃清洗胶体小球的模 板后, 如图 6c 所示, 明显有大量的小球被完全包裹在丝 素蛋白形成的凝胶中无法被溶解清洗掉. 因此, 我们猜 测，最终小球无序排列的原因是由于丝素蛋白分子与胶 体小球之间有较强的亲和力, 从而抑制了小球与小球之 间的相互作用，进而削弱了小球的自组装效应.
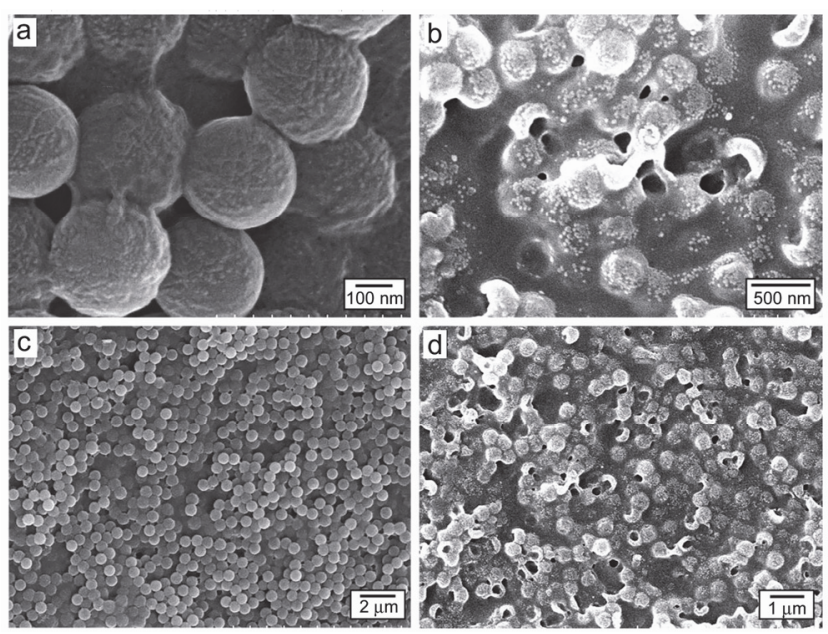

图 6 聚苯乙烯微球和丝素蛋白二元共聚自组装形成结构的 SEM 图 像

Figure 6 SEM images of polystyrene sphere-silk fibroin system co-assembly structures

(a, b) Co-assembly structure before sacrificing template, (c, d) Co-assembly structures after PS elimination

\section{4 丝素蛋白分子与胶体微球的相互作用研究}

为了进一步确认丝素蛋白分子与聚苯乙烯微球表 面的相互作用阻碍了微球的有序自组装, 本文将丝素蛋 白/聚苯乙烯微球共混 $30 \mathrm{~min}$ 后进行反复离心处理, 取 重新分散的悬浮液滴加在洗干净的硅片或者玻璃片上， 待样品干燥后分别用扫描电镜和荧光共聚焦显微镜观 察. 从图 7a 可以看出, 纯的聚苯乙烯微球表面非常光 滑. 与蚕丝共混后, 若胶体小球和丝素蛋白没有相互作 用，游离的丝素蛋白分子在反复离心过程中会被分离出 去. 而我们的扫描图像图 $7 \mathrm{~b}$ 可以看到小球表面不再光

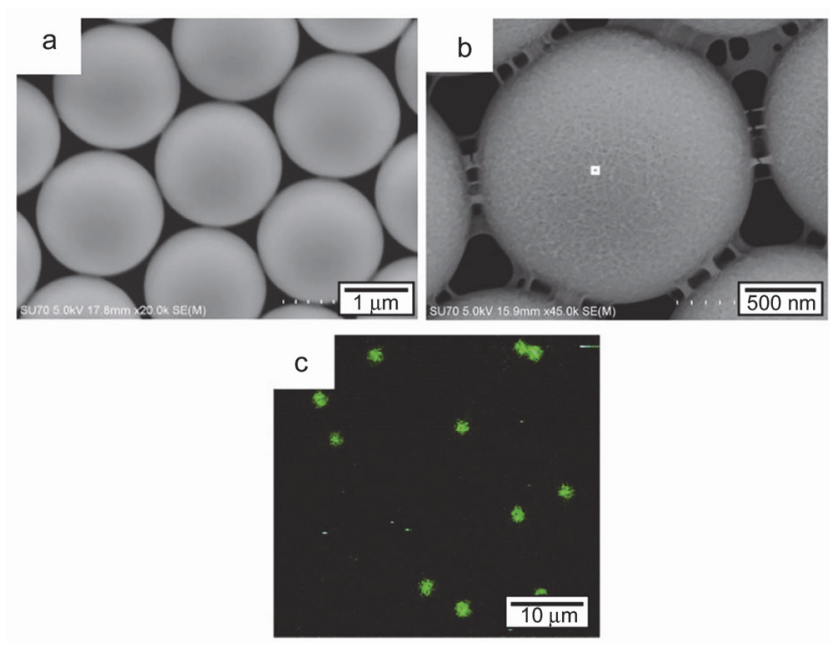

图 7 (a)未经丝素蛋白处理的聚苯乙烯微球在纯水条件下的组装和 (b)被丝素蛋白生长过聚苯乙烯微球的自组装的 SEM 图像及(c)在被 FITC 修饰的丝素蛋白溶液中浸泡过的聚苯乙烯微球的苂光图像

Figure 7 SEM images of self-assembly of (a) original polystyrene nanoparticles and (b) polystyrene nanoparticles incubated by silk and (c) fluorescent image of polystyrene nanoparticles incubated by FITC-labeled silk fibroin 
滑, 而是变得十分毛䊁. 这表明丝素蛋白与胶体小球具 有一定的相互作用, 仍然有大量的丝素蛋白在胶体小球 的表面富集. 苂光共聚焦图像 7c 也可以看到 PS 小球包 覆了一层 FITC 修饰的丝素蛋白. 此外, 根据已有文献 的研究 ${ }^{[25]}$, 胶体小球表面的羟基和丝素蛋白的氨基会 产生氢键相互作用，二者的相互作用使得丝素蛋白分子 极为容易在含有羟基的 PS 微球表面附着, 并发生异相 生长. 综上, 我们认为大分子丝素蛋白与胶体小球表面 产生较强作用, 二者的相互作用对胶体小球的组装有抑 制作用，从而不能通过与胶体小球产生有序的共组装来 制备无缺陷反蛋白石薄膜.

\section{5 共组装法的优势与局限性}

如图 8 所示, 与传统的牺牲模板法相比, 基质前驱 体溶液与聚苯乙烯小球共组装策略具有两个十分明显 的优点. 第一, 在制备反蛋白石薄膜上缩短了操作流程, 提高了效率. 第二, 共组装法可以有效避免缺陷的产生. 例如, 我们已成功运用共组装法合成大面积无缺陷的二 氧化硅反蛋白石薄膜. 但是共组装法也有一定的局限 性. 为了让小球能在基质前驱体溶液中有序的组装, 需
要避免选取与胶体小球有较大作用力的基质. 也就是 说，该方法不适用于基质前驱体与胶体小球有较强相互 作用的体系. 例如丝素蛋白一胶体小球共组装体系，由 于胶体小球和丝素蛋白具有较强的相互作用，会促使丝 素蛋白在胶体小球表面快速吸附，阻碍小球和小球之间 自身的组装效应.

\section{3 结论}

探讨了将胶体小球分散在两种基质前驱体(TEOS 前驱体溶液和丝素蛋白再生溶液)中进行共组装, 进而 除去模板制备无裂痕反蛋白石结构薄膜的可行性. 结果 证明，TEOS 前驱体自身的溶胶凝胶转变不会明显影响 到胶体小球的有序自组装. 通过选取合适浓度的 TEOS 前驱体和 PS 微球混合悬浮液, 可以直接共组装得到内 部小球排列规整的薄膜，再去除微球模板即可得到大面 积无缺陷的二氧化硅反蛋白石薄膜. 而大分子丝素蛋白 与胶体小球有着较强的亲和力，易附着在胶体小球表 面，该过程会抑制胶体小球之间有序的自组装，不适用 于大面积无缺陷反蛋白石薄膜的制备. 本实验说明，选

(a) Indirect approach through template self-assembly and matrix infiltration

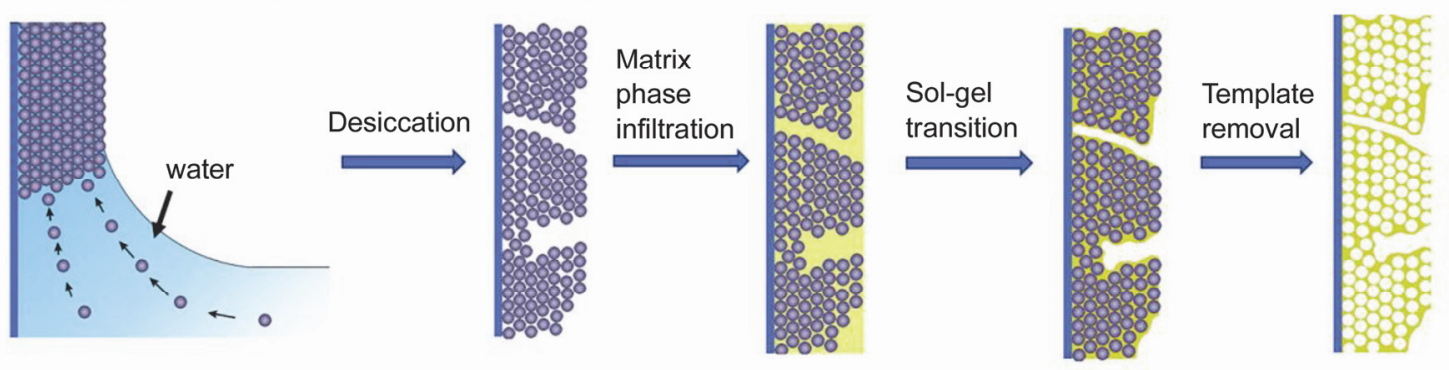

(b) Direct template/TEOS co-assembly
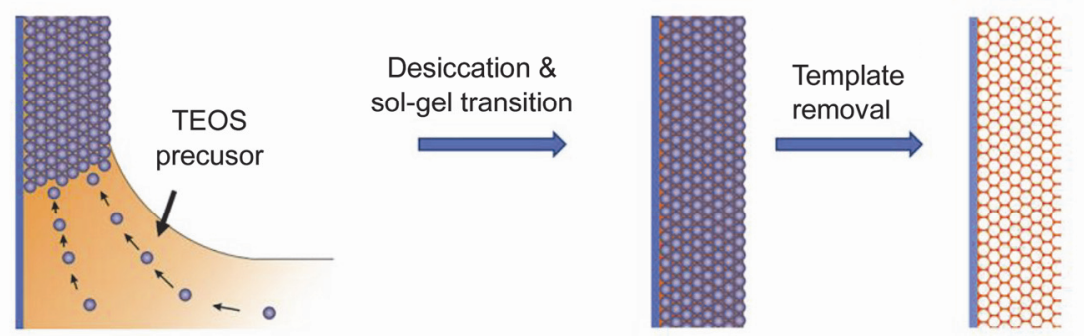

(c) Direct template/silk fibroin co-assembly
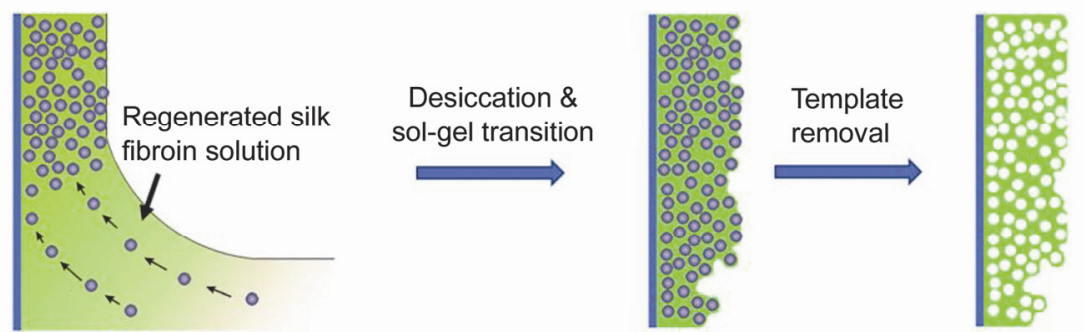

图 8 (a)反蛋白石结构光子晶体的传统的制备方法和(b)正硅酸乙酯和聚苯乙烯微球共同组装的示意图及(c)丝素蛋白和聚苯乙烯微球共同组装的 示意图

Figure 8 (a) Schematic illustration of "conventional" colloidal template self-assembly for the fabrication of inverse-opal films, (b) co-assembly of TEOS $\&$ polystyrene nanoparticles, and (c) co-assembly of silk fibroin \& polystyrene nanoparticles 
择合适的二元体系进行共组装能简化操作流程, 可以得 到大面积、无缺陷的反蛋白石薄膜. 该方法为制备性能 更好的反蛋白石结构材料提供了新的途径, 相信通过该 方法所优化制备的无裂痕反蛋白石薄膜能被更广泛应 用到各个相关领域.

\section{4 实验部分}

\section{1 仪器与试剂}

箱式电阻炉： sx2-4-10, 上海一恒科技有限公司. SEM 图像: SU-70 扫描电镜, 样品表面喷金处理; 反射 光谱: 海洋光学 USB2000+光纤光谱仪; 苂光图像: 莱 卡 Leica TCS SP8 共聚焦激光显微镜. 碳酸氢钠: 分析 纯, 上海国药集团化学试剂有限公司; 溴化锂: 分析纯, 上海阿拉丁生化科技股份有限公司; 苯乙烯: 化学纯, 广东汕头市西陇化工; 无水乙醇: 分析纯, 广东汕头市 西陇化工; 氢氧化钠: 化学纯, 广东汕头市西陇化工; 浓硫酸: 分析纯，广东汕头市西陇化工; $30 \%$ 过氧化氢: 分析纯, 广东汕头市西陇化工; 过硫酸铵: 分析纯, 广 东汕头市西陇化工; 丙烯酸: 分析纯, 广东汕头市西陇 化工; 正硅酸乙酯: 分析纯, 广东汕头市西陇化工; 盐 酸: 分析纯 $36 \% \sim 38 \%$, 广东汕头市西陇化工; 四氢呋 喃: 分析纯, 广东汕头市西陇化工.

\section{2 聚苯乙烯微球的制备}

按文献[17]中的方法, 采用无皇乳液聚合法制备单 分散的聚苯乙烯(PS)微球, 具体的制备过程如下. 首先, 量取 $300 \mathrm{~mL}$ 超纯水, $38.5 \mathrm{~mL}$ 苯乙烯 $(\mathrm{St})$ 和 $0 \sim 333 \mu \mathrm{L}$ 丙 烯酸(AA)加入四口烧瓶, 密封, 通氮气, 充分搅拌 20 $\min$ 后开始加热. 温度达到 $70{ }^{\circ} \mathrm{C}$ 并稳定后, 再将 $10 \mathrm{~mL}$ 配置好的 $17.5 \mathrm{mg} / \mathrm{mL}$ 引发剂过硫酸铵全部注入烧瓶内, 继续反应 $7 \mathrm{~h}$. 最后, 将反应完成液用定量滤纸过滤后 离心 3 次, 去掉上清液, 加入超纯水溶解后低温存储. 通过电位粒度仪(Mastersizer 2000)检测, 试验中所用的 PS 微球多分散性指数 PDI 均小于 0.01 .

\section{3 丝素蛋白再生溶液的制备}

取适量虫茧置于 $0.5 \mathrm{wt} \%$ 的碳酸氢钠水溶液中, 煮 沸并配合搅拌处理脱胶 $30 \mathrm{~min}$, 用清水煮沸漂洗 30 min. 将上述步骤重复操作两次, 再用清水煮沸漂洗两 次后置于烘箱中, 在 $60{ }^{\circ} \mathrm{C}$ 下烘干 $24 \mathrm{~h}$. 将脱胶丝素蛋 白溶解于过滤后的 $9.4 \mathrm{~mol} \cdot \mathrm{L}^{-1}$ 溴化锂水溶液中, 放置在 $60{ }^{\circ} \mathrm{C}$ 的烘箱中密封溶解 $4 \mathrm{~h}$ 后, 将溶液装入截留分子量 为 $3500 \mathrm{D}$ 的透析袋中, 用去离子水进行透析处理 $48 \mathrm{~h}$, 即得到透明的丝素蛋白溶液 ${ }^{[26,27]}$.

\section{4 正硅酸乙酯与聚苯乙烯微球的共组装}

配置正硅酸乙酯(TEOS)的前驱体溶液: 将正硅酸 乙酯、 $0.1 \mathrm{~mol} \cdot \mathrm{L}^{-1}$ 的盐酸和无水乙醇以质量比 $2: 2: 3$ 的比例混合, 并在室温下摚拌 $1 \mathrm{~h}$ 以备使用. 配置 $0.075 \sim 0.5 \mathrm{wt} \%$ 的 PS 小球悬浮液. 取 $20 \mathrm{~mL} \mathrm{PS}$ 小球悬
浮液放入玻璃瓶中, 并加入 $0.15 \mathrm{~mL}$ 正硅酸乙酯前驱体 溶液. 将 $1 \mathrm{~cm} \times 4 \mathrm{~cm}$ 的玻璃片坚直插入上述混合溶液 的玻璃瓶中. 之后, 将玻璃瓶静置于 $65{ }^{\circ} \mathrm{C}$ 的烘箱里, 待干燥挥发后，取出玻璃片，并放在高温电阻箱中， $2 \mathrm{~h}$ 内升温至 $500{ }^{\circ} \mathrm{C}$, 保持 $2 \mathrm{~h}$ 后, 待其缓慢降温至室温, 即可得到具有反蛋白石结构的二氧化硅薄膜.

\section{5 丝素蛋白与聚苯乙烯微球的共组装}

配置浓度为 $0.1 \mathrm{wt} \%$ 的 PS 小球悬浮液, 并与浓度为 $0.4 \mathrm{wt} \%$ 的丝素蛋白溶液以 $1: 1$ 的比例混合, 取 $20 \mathrm{~mL}$ 共混溶液放入玻璃瓶中, 将 $1 \mathrm{~cm} \times 4 \mathrm{~cm}$ 的玻璃片坚直 插入装有上述混合溶液的玻璃瓶中，并放置在 $60{ }^{\circ} \mathrm{C}$ 的 烘箱中干燥 $24 \mathrm{~h}$. 干燥挥发后, 取出玻璃片放在四氢呋 喃溶液中，浸泡 $1 \mathrm{~h}$ 后取出，用去离子水反复冲洗，即可 得到丝素蛋白的多孔薄膜.

\section{6 丝素蛋白分子的苂光标记}

将 $1 \mathrm{~mL}$ 浓度为 $0.5 \mathrm{~mol} / \mathrm{mL}$ 的碳酸钠溶液与 $10 \mathrm{~mL}$ 浓度为 $70 \mathrm{mg} / \mathrm{mL}$ 的丝素蛋白溶液混合. 配置浓度为 5 $\mathrm{mg} / \mathrm{mL}$ 的异硫氰(酸苂光素(FITC)的二甲基亚砜(DMSO) 溶液, 取 $1.4 \mathrm{~mL}$ 缓缓加入上述已经配置好的丝素蛋白 混合溶液. 在暗室中搅拌 $2 \mathrm{~h}$ 后, 用清水透析 $6 \mathrm{~h}$, 即可 获得被 FITC 标记的丝素蛋白溶液.

\section{7 丝素蛋白与聚苯乙烯微球相互作用研究}

在研究丝素蛋白与聚苯乙烯相互作用时, 为方便 SEM 和苂光共聚焦观察，我们在该部分实验选用 $2 \mu \mathrm{m}$ 的 PS 微球. 取经过离心再分散处理过的 $200 \mu \mathrm{L}$ 浓度为 $0.01 \mathrm{~g} / \mathrm{mL}$ 的 PS 微球单分散溶液, 与 $1 \mathrm{~mL}$ 浓度为 5 $\mathrm{mg} / \mathrm{mL}$ 未修饰或 FITC 修饰过的丝素蛋白溶液混合. 待 丝素蛋白分子与 PS 微球混合上 $30 \mathrm{~min}$ 后，通过多次离 心处理，除去混合溶液中游离的丝素蛋白分子，将沉淀 再分散到 $1 \mathrm{~mL}$ 的超纯水中, 制成悬浮液. 分别取适量 纯净的 PS 微球悬浮液和与丝素蛋白作用过的 PS 微球悬 浮液滴涂在硅片或者玻璃片上，待干燥后，通过 SEM和 苂光共聚焦表征来研究分析小球与丝素蛋白的相互作 用情况.

\section{References}

[1] Whitesides, G. M.; Grzybowski, B. Science 2002, 295, 2418

[2] Zhao, X.; Su, F.; Yan, Q.; Guo, W.; Bao, X. Y.; Lv, L.; Zhou, Z. J. Mater. Chem. 2006, 16, 637.

[3] Holland, B. T.; Blanford, C. F.; Stein, A. Science 1998, 281, 538.

[4] Arsenault, A. C.; Clark, T. J.; von Freymann, G.; Cademartiri, L.; Sapienza, R.; Bertolotti, J.; Vekris, E.; Wong, S.; Kitaev, V.; Manners, I. Nat. Mater. 2006, 5, 179.

[5] Rinne, S. A.; García-Santamaría, F.; Braun, P. V. Nat. Photonics 2008, 2, 52 .

[6] Choi, S. W.; Xie, J.; Xia, Y. Adv. Mater. 2009, 21, 2997.

[7] Lee, K.; Asher, S. A. J. Am. Chem. Soc. 2000, 122, 9534.

[8] Hatton, B.; Mishchenko, L.; Davis, S.; Sandhage, K. H.; Aizenberg, J. Proc. Natl. Acad. Sci. U. S. A. $2010,107,10354$.

[9] Lytle, J. C.; Stein, A. Annual Review of Nano Research, Vol. 1, Eds.: Cao, G. Z.; Brinker, C. J., World Scientific Publishing Co., Singapore, 2006, 1 , pp. $1 \sim 14$.

[10] Velev, O. D.; Lenhoff, A. M. Curr. Opin. Colloid. Interface Sci. 
$\mathbf{2 0 0 0 , 5 , 5 6 . ~}$

[11] Li, Y.; Qi, L.-M. Acta Chim. Sinica 2015, 73, 869. (李扬; 齐利民, 化学学报, 2015, 73, 869.)

[12] Jiang, F.-G.; Yao, J.-R.; Chen, X.; Shao, Z.-Z. Acta Chim. Sinica 2009, 67, 1675. (蒋伏广, 姚晋荣, 陈新, 邵正中, 化学学报, 2009, 67, 1675.)

[13] Tu, H.; Yu, R.; Lin, Z.; Zhang, L.; Lin, N.; Yu, W. D.; Liu, X. Y. Adv. Funct. Mater. 2016, 26, 9032.

[14] Ke, G.-Z.; Xie, H.-F.; Ruan, R.-P.; Yu, W.-D. Energy Convers. Manage. 2010, 51, 2294.

[15] Liu, R.; Wan, L.; Liu, S.; Pan, L.; Wu, D.; Zhao, D. Adv. Funct. Mater. 2015, 25, 526

[16] Schroden, R. C.; Al-Daous, M.; Blanford, C. F.; Stein, A. Chem. Mater. 2002, 14, 3305.

[17] Diao, Y. Y.; Liu, X. Y.; Toh, G. W.; Shi, L.; Zi, J. Adv. Funct. Mater. 2013, 23, 5373.

[18] Wong, S.; Kitaev, V.; Ozin, G. A. J. Am. Chem. Soc. 2003, 125,
15589.

[19] Zhou, Z.; Zhao, X. Langmuir 2005, 21, 4717.

[20] Zhang, T. H.; Kuipers, B. W.; Groenewold, J.; Kegel, W. K. Soft Matter. 2015, 11, 297.

[21] Zhang, T. H.; Liu, X. Y. Chem. Soc. Rev. 2014, 43, 2324

[22] Chabanov, A. A.; Jun, Y.; Norris, D. J. Appl. Phys. Lett. 2004, 84, 3573 .

[23] Huang, Y.; Zhou, J.; Su, B.; Shi, L.; Jiang, L. J. Am. Chem. Soc. 2012, 134, 17053 .

[24] Busch, K.; John, S. Phys. Rev. E 1998, 58, 3896.

[25] Chen, Z.; Zhang, H.; Lin, Z.; Lin, Y.; van Esch, J. H.; Liu, X. Y. Adv. Funct. Mater. 2016, 26, 8978.

[26] Nagarkar, S.; Nicolai, T.; Chassenieux, C.; Lele, A. Phys. Chem. Chem. Phys. 2010, 12, 3834.

[27] Cao, H.; Chen, X.; Shao, Z.-Z. Acta Chim. Sinica 2008, 66, 2059. (曹惠, 陈新, 邵正中, 化学学报, 2008, 66, 2059.) 